\title{
DISCRETE VELOCITY MODELS: A STUDY OF THE HYDRODYNAMIC LIMIT
}

\author{
Hans Babovsky \\ Technische Universität Ilmenau \\ D-98684 Ilmenau, Germany \\ e-mail: hans.babovsky@tu-ilmeau.de
}

Keywords: Discrete Velocity Models, diffusive scaling, hydrodynamic limit, closure relations.

\begin{abstract}
We investigate Discrete Velocity Models as a numerical tool for the simulation of rarefied flows in the hydrodynamic limit. The study is based on the analysis of the bifurcation structure of a related linearized transport operator. In combination with a meaningful scaling this produces explicit formulas for the transport coefficients and closure relations depending on details of the model collision operator. The theory is applicable even in cases when the classical asymptotic analysis approach fails or leads to physically irrelevant results [7, 8].
\end{abstract}




\section{INTRODUCTION}

Discrete Velocity Models (DVM) are a promising alternative to the commonly used DSMC methods for the numerical simulation of rarefied gas flows. While the latter should keep for the next time their prominent position as long as real gas effects are concerned, the first ones are able to provide new insights into questions e.g. concerning the hydrodynamic limit. This is on one hand due to the impressing development of powerful yet achievable hard ware tools (GPU, GPU). On the other hand have recent contributions taught how to interpret DVMs as numerical integration tools for the Boltzmann collision operator $[1,2]$ which allows to tune such models with respect to order of consistency and to numerical efficiency [3].

In [4] a formalism was introduced for DVM's based on two-particle collisions which provides an analytic expression for the pseudo inverse of the linearized collision operator and allows to calculate Navier-Stokes corrections to the Euler equations in the hydrodynamic limit. In this context it was argued why at least small velocity grids can reveal an appropriate macroscopic description only in the case of small Mach numbers. In the present paper we study the behaviour of DVM's at small bulk velocities. We identify a bifurcation phenomenon of the linearized steady transport operator when perturbing the Mach zero Maxwellian. This feature yields an appropriate macroscopic description when applying the correct scaling limit. The approach is constructive in the sense that it provides explicit formulas for hydrodynamic flow parameters and closure relations for the moment hierarchy. In terms of an evaporation condensation problem for a binary gas mixture we shortly discuss the difference between the present approach and some standard asymptotic analysis techniques.

Though the following framework is applicale to three-dimensional velocity grids, we restrict for sake of brevity to the two-dimensional case.

\section{THE COLLISION MODEL}

We consider a DVM on a finite integer grid $\mathcal{V}=\left\{\mathbf{v}_{1}, \ldots, \mathbf{v}_{N}\right\} \subset \mathbb{R}^{2}, \mathbf{v}_{i}=\left(v_{x}^{(i)}, v_{y}^{(i)}\right)$. The collision operator is composed of single collision events described a quadruple

$$
\alpha=(i, j, k, l) \doteq\left(\mathbf{v}_{i}, \mathbf{v}_{j}, \mathbf{v}_{k}, \mathbf{v}_{l}\right) \in \mathcal{V}^{4}
$$

and the single-collision operator $J_{\alpha}$ for a density $\mathbf{f}=\left(f_{r}\right)_{r=1}^{N} \doteq(f(\mathbf{v}), \mathbf{v} \in \mathcal{V})$ given by

$$
J_{\alpha}[\mathbf{f}, \mathbf{f}]=\left(f_{k} f_{l}-f_{i} f_{j}\right) \cdot \mathbf{s}_{\alpha}, \quad \mathbf{s}_{\alpha}=\left(\mathbf{e}_{i}+\mathbf{e}_{j}-\mathbf{e}_{k}-\mathbf{e}_{l}\right)
$$

with $\mathbf{e}_{r}=\mathrm{r}$-th canonical unit vector. (For a more detailed introduction into this formalism see [4].) $J_{\alpha}$ satisfies the physical conservation laws for mass, momenta and energy, iff $\overline{\mathbf{v}_{i} \mathbf{v}_{j}}$ and $\overline{\mathbf{v}_{k} \mathbf{v}_{l}}$ are the diagonals of a rectangle in $\mathbb{R}^{2}$. The set of quadruples $\alpha$ with this property is denoted by $\mathcal{R}$. The full collision operator reads

$$
J[\mathbf{f}, \mathbf{f}]=\sum_{\alpha \in \mathcal{R}} \gamma_{\alpha} \cdot J_{\alpha}[\mathbf{f}, \mathbf{f}]
$$

with collision frequencies $\gamma_{\alpha} \geq 0$. We assume the set of $\alpha$ with $\gamma_{\alpha}>0$ large enough such that the besides mass, momenta and energy there are no other invariants. It is well-known that 
in this case the classical $H$-Theorem is satisfies, and as a consequence the set of equilibrium functions of $J$ is equal to the set of Maxwellians

$$
\mathbf{f}=\mathbf{f}[\rho, \overline{\mathbf{v}}, \Theta]=\left(\rho \exp \left(-\frac{|\mathbf{v}-\overline{\mathbf{v}}|^{2}}{2 \Theta}\right), \mathbf{v} \in \mathcal{V}\right)
$$

The linearization of $J$ around a Maxwellian $\mathbf{f}$ is given by the operator $J[\mathbf{f}+\phi, \mathbf{f}+\phi]$ neglecting the terms quadratic in $\phi$. Taking into account the identity $f_{i} f_{j}=f_{k} f_{l}=: \phi_{\alpha}$ for $\alpha=(i, j, k, l) \in \mathcal{R}$ and defining the matrices

$$
P_{\alpha}=\left(\mathbf{e}_{i}, \mathbf{e}_{j}, \mathbf{e}_{k}, \mathbf{e}_{l}\right), \quad \Gamma=\mathbb{1}_{ \pm} \cdot \mathbb{1}_{ \pm}^{T}, \quad \mathbb{1}_{ \pm}=(1,1,-1,-1)^{T}
$$

the linearization takes the form

$$
J[\mathbf{f}, \phi]+J[\phi, \mathbf{f}]=C F^{-1}
$$

with $F=\operatorname{diag}\left(f_{i}, i=1 \ldots N\right)$ and

$$
C=-\sum_{\alpha \in \mathcal{R}} \pi_{\alpha} P_{\alpha} \Gamma P_{\alpha}^{T}, \quad \pi_{\alpha}=\gamma_{\alpha} \phi_{\alpha}
$$

The main properties of $C$ are easy to prove [4] and given as follows.

Lemma: $C$ is symmetric and negative semidefinite. The geometric zero eigenspace has dimension 4 and is given by

$$
\mathcal{M}=\operatorname{span}\left(\mathbb{1}=(1, \ldots, 1)^{T}, \mathbf{v}_{x}=\left(v_{x}^{(i)}\right)_{i=1}^{N}, \mathbf{v}_{y}=\left(v_{y}^{(i)}\right)_{i=1}^{N},|\mathbf{v}|=\left(\left|\mathbf{v}_{i}\right|^{2}\right)_{i=1}^{N}\right)
$$

Much of the following is based on symmetry arguments. We assume the grid $\mathcal{V}$ and the collision model on $\mathcal{V}$ to be invariant with respect to reflections at $v_{x}=0$ resp. $v_{y}=0$. Precisely this means the following. Given $\mathbf{v}=\left(v_{x}, v_{y}\right) \in \mathcal{V}$, define $T_{x} \mathbf{v}=\left(-v_{x}, v_{y}\right)$ and $T_{y}=\left(v_{x},-v_{y}\right) . T_{x}$ and $T_{y}$-invariance of $\mathcal{V}$ means that

$$
\mathbf{v} \in \mathcal{V} \quad \Leftrightarrow \quad T_{x} \mathbf{v} \in \mathcal{V} \quad \Leftrightarrow \quad T_{y} \mathbf{v} \in \mathcal{V}
$$

Given $\mathbf{f} \in \mathbb{R}^{N}$ (as a function on $\mathcal{V}$ ), define $T_{x} \mathbf{f} \in \mathbb{R}^{N}$ by $T_{x} \mathbf{f}(\mathbf{v})=\mathbf{f}\left(T_{x} \mathbf{v}\right)$, and similarly $T_{y} \mathbf{f}$. $T_{x^{-}}$and $T_{y}$-invariance of the collision operator $J[\mathbf{f}, \mathbf{f}]$ means that $J\left[T_{x} \mathbf{f}, T_{x} \mathbf{f}\right]=T_{x} J[\mathbf{f}, \mathbf{f}]$ and $J\left[T_{y} \mathbf{f}, T_{y} \mathbf{f}\right]=T_{y} J[\mathbf{f}, \mathbf{f}]$.

$\mathbf{f}$ is called $T_{x}$-even, if $\mathbf{f}=T_{x} \mathbf{f}$, and $T_{x}$-odd, if $\mathbf{f}=-T_{x} \mathbf{f}$. Similarly, $T_{y}$-even and $T_{y}$-odd are defined.

\section{STEADY PROBLEMS AND BIFURCATION}

Consider the steady one-dimensional problem

$$
v_{x} \partial_{x} f=J[f, f]
$$

on $[-1,1]$ with boundary conditions at $x= \pm 1$. In the case of totally reflecting boundaries, the flux into wall direction is zero. Consider a small perturbation $\mathrm{f}_{0}+\phi, \phi \perp F_{0} \mathcal{M}$, around a centered Maxwellian

$$
\mathbf{f}_{0}(v)=\mathbf{f}[\rho, 0, \Theta](v)
$$


Neglecting the terms quadratic in $\phi$ yields the linearized system

$$
v_{x} \partial_{x} \phi=J\left[\mathbf{f}_{0}, \phi\right]+J\left[\phi, \mathbf{f}_{0}\right]=C F_{0}^{-1} \phi
$$

We assume that $\mathcal{V}$ contains no velocities with $v_{x}=0$. In this case, because of $T_{x^{-}}$ invariance, $N=2 n$ has to be even. We choose a numbering of the velocities $\mathbf{v}_{i}=\left(v_{x}^{(i)}, v_{y}^{(i)}\right)$ such that $v_{x}^{(i)}>0$, and $\mathbf{v}_{n+i}=T_{x} \mathbf{v}_{i}$ for $i=1, \ldots, n$. Under this restriction, the linearized equation represents an ODE system

$$
\partial_{x} \phi=L_{0} \phi
$$

Under reasonable conditions on the collision model (see [5]), the linearized collision operator

$$
L_{0}=V^{-1} C F_{0}^{-1}
$$

has a Jordan normal form given by

$$
N=\operatorname{diag}\left(0,0, N_{0}, N_{0}, \Lambda,-\Lambda\right)
$$

with a positive diagonal matrix $\Lambda=\operatorname{diag}\left(\lambda_{1} \ldots \lambda_{p}\right)$ and the Jordan block

$$
N_{0}=\left(\begin{array}{ll}
0 & 1 \\
0 & 0
\end{array}\right)
$$

The columns $\mathbf{t}_{i}^{(0)}$ of the corresponding transformation matrix $T^{(0)}$ are characterized as follows.

- $\mathbf{t}_{1}^{(0)}=F_{0} v_{x}$ is $T_{x}$-odd and zero-eigenvector of $L$.

- $\operatorname{span}\left\{\mathbf{t}_{2}^{(0)}, \mathbf{t}_{3}^{(0)}\right\}=F_{0} \cdot \operatorname{span}\left\{\mathbb{1},|v|^{2}\right\}$; in particular, $\mathbf{t}_{2}^{(0)}$ and $\mathbf{t}_{3}^{(0)}$ are $T_{x}$-even zero-eigenvectors of $L_{0}$.

- $\mathbf{t}_{4}^{(0)}$ is $T_{x}$-odd and solution of $L_{0} \mathbf{t}_{4}^{(0)}=\mathbf{t}_{3}^{(0)}$.

- $\mathbf{t}_{5}^{(0)}=F_{0} v_{y}$ is $T_{x}$-even and $T_{y}$-odd and zero-eigenvector of $L$.

- $\mathbf{t}_{6}^{(0)}$ is $T_{x^{-}}$and $T_{y^{-}}$odd and solution of $L_{0} \mathbf{t}_{6}^{(0)}=\mathbf{t}_{5}^{(0)}$.

- $L_{0} \mathbf{t}_{6+i}^{(0)}=\lambda_{i} \mathbf{t}_{6+i}^{(0)}, i=1, \ldots, p$.

- $L_{0} \mathbf{t}_{6+p+i}^{(0)}=-\lambda_{i} \mathbf{t}_{6+p+i}^{(0)}, i=1, \ldots, p$.

Thus the general solution $\phi$ of the ODE system is

$$
\begin{aligned}
\phi(x)= & c_{4}\left(\mathbf{t}_{4}^{(0)}+x \cdot \mathbf{t}_{3}^{(0)}\right)+c_{5}\left(\mathbf{t}_{6}^{(0)}+x \cdot \mathbf{t}_{5}^{(0)}\right) \\
& +\exp ((1+x) \Lambda) \phi_{+}(-1)+\exp ((1-x) \Lambda) \phi_{-}(+1)
\end{aligned}
$$

The sum $F=c_{4} \mathbf{t}_{4}^{(0)}+c_{6} \mathbf{t}_{6}^{(0)}$ is the socalled fluctuation part which is mapped to the zero eigenspace of $L$. The constant gradient part $x \cdot\left(c_{4} \mathbf{t}_{3}^{(0)}+c_{6} \mathbf{t}_{5}^{(0)}\right)$ is called the macroscopic part, while the last two terms represent the boundary layers. From now on we restrict to $T_{y}$-even solutions, putting $c_{5}=0$. 
The solvability condition for $L_{0} \mathbf{t}_{4}^{(0)}=\mathbf{t}_{3}^{(0)}$ is that $\mathbf{t}_{3}^{(0)}$ is orthogonal to the kernel of $L_{0}^{T}$, in particular to $\mathbf{v}_{x}^{2}$. This means that we may choose $\mathbf{t}_{3}^{(0)}=F_{0}\left(\mathbb{1}+\xi \cdot|\mathbf{v}|^{2}\right)$ with

$$
\xi=\frac{\left\langle\mathbf{v}_{x}^{2}, f_{0}\right\rangle}{\left\langle\mathbf{v}_{x}^{2}, f_{0}|\mathbf{v}|^{2}\right\rangle}
$$

We now introduce a small shift by changing the Maxwellian into

$$
f_{\delta}(\mathbf{v})=\exp \left(-\left|\mathbf{v}-\delta \cdot \mathbf{e}_{x}\right|^{2} / 2 \Theta\right)
$$

and the corresponding matrix $F_{0}$ into

$$
F_{\delta}(\mathbf{v})=\operatorname{diag}\left(f_{\delta}(\mathbf{v}), \mathbf{v} \in \mathcal{G}\right)=F_{0} \cdot\left(I+\frac{\delta}{\Theta} V_{x}\right)+\mathcal{O}\left(\delta^{2}\right)
$$

In the following we investigate the bifurcation structure of the zero eigenspace. Thus we are interested only in infinitesimal shifts and restrict an $\mathcal{O}(\delta)$ theory. The linearized collision operator $L_{0}$ is affected twofold by this change and takes the form

$$
L_{\delta} \doteq V_{x}^{-1}\left(C+\delta \cdot C^{\prime}\right)\left(F_{0}+\delta \cdot F^{\prime}\right)^{-1} \doteq L_{0}+\delta \cdot V_{x}^{-1} C^{\prime} F_{0}-\delta \cdot V_{x}^{-1} C F^{\prime}
$$

The first part of the perturbation, $\delta \cdot V_{x}^{-1} C^{\prime} F_{0}$ stems from the change of the factor $\phi_{\alpha}$ in (5). This part does not contribute to the bifurcation structure and is neglected here. Thus we obtain as the new linearized collision operator

$$
L_{\delta} \doteq L_{0} \cdot\left(I-\frac{\delta}{\Theta} V_{x}\right)
$$

The perturbation changes the nullspace into

$$
F_{\delta} \mathcal{M} \doteq F_{0}\left(I+\frac{\delta}{\Theta} V_{x}\right) \mathcal{M}
$$

The eigenspace data corresponding to nonzero eigenvectors suffer an analytic change and do not influence the qualitative structure of the Jordan normal form. The main change results from the non-solvability of the equation $L_{\delta} \mathbf{t}_{4}^{\delta}=\mathbf{t}_{3}^{\delta}$. It has to be replaced with the equation

$$
L_{\delta} \mathbf{t}_{4}^{\delta}=\mathbf{t}_{3}^{\delta}+\frac{\delta \cdot \lambda}{\Theta} \cdot \mathbf{t}_{4}^{\delta}
$$

The choice

$$
\mathbf{t}_{3}^{\delta}=\left(I+\frac{\delta}{\Theta} V_{x}\right) \mathbf{t}_{3}^{0}+\frac{\delta \cdot \mu}{\Theta} V_{x} \mathbf{f}_{0}
$$

and the ansatz $\mathbf{t}_{4}^{\delta}=\mathbf{t}_{4}^{0}+\delta \Theta^{-1} \mathbf{t}_{4}^{\prime}$ leads to the equation

$$
L_{0} \mathbf{t}_{4}^{\prime}=V_{x} \mathbf{t}_{3}^{0}+L_{0} V_{x} \mathbf{t}_{4}^{0}+\lambda \mathbf{t}_{4}^{0}+\mu V_{x} \mathbf{f}_{0}
$$


for the $T_{x}$-even function $\mathbf{t}_{4}^{\prime}$ with the solvability condition

$$
\left(\begin{array}{cc}
\left\langle\mathbf{v}_{x}, \mathbf{t}_{4}^{0}\right\rangle & \left\langle\mathbf{v}_{x}^{2}, \mathbf{f}_{0}\right\rangle \\
\left\langle\mathbf{v}_{x}|\mathbf{v}|^{2}, \mathbf{t}_{4}^{0}\right\rangle & \left\langle\mathbf{v}_{x}^{2}|\mathbf{v}|^{2}, \mathbf{f}_{0}\right\rangle
\end{array}\right)\left(\begin{array}{c}
\lambda \\
\mu
\end{array}\right)=-\left(\begin{array}{c}
\left\langle\mathbf{v}_{x}^{2}, \mathbf{t}_{3}^{0}\right\rangle+\left\langle\mathbf{v}_{x}, L_{0} V_{x} \mathbf{t}_{4}^{0}\right\rangle \\
\left\langle\mathbf{v}_{x}^{2}|\mathbf{v}|^{2}, \mathbf{t}_{3}^{0}\right\rangle+\left\langle\mathbf{v}_{x}|\mathbf{v}|^{2}, L_{0} V_{x} \mathbf{t}_{4}^{0}\right\rangle
\end{array}\right)
$$

for $(\lambda, \mu)$.

As a consequence, choosing $\bar{v}:=\delta \Theta^{-1}$, the corresponding Jordan block $N_{0}$ changes into

$$
N_{\delta}=\left(\begin{array}{cc}
0 & 1 \\
0 & \lambda \bar{v}
\end{array}\right)
$$

which is similar to the diagonal matrix $\operatorname{diag}(0, \lambda \bar{v})$. The following result is an immediate consequence of this.

Theorem: (a) The solution of the perturbed ordinary differential system takes the form

$$
\begin{aligned}
\phi(x)= & c_{4} \cdot\left(\exp (\lambda \bar{v} x) \mathbf{t}_{4}+\frac{\exp (\lambda \bar{v} x)-1}{\lambda \bar{v}} \cdot \mathbf{t}_{3}\right) \\
& +\exp \left((1+x) \Lambda_{\delta}^{+}\right) \phi_{+}(-1)+\exp \left((1-x) \Lambda_{\delta}^{-}\right) \phi_{-}(+1)
\end{aligned}
$$

(b) The fluxes of moment vectors $\mathbf{m} \in \mathcal{M}$ are constant and given by

$$
\left\langle\mathbf{v}_{x} \mathbf{m}, \phi\right\rangle=c_{4}\left\langle\mathbf{v}_{x} \mathbf{m}, \mathbf{t}_{4}^{\delta}\right\rangle
$$

\section{DIFFUSIVE SCALING AND CLOSURE RELATIONS}

In order to derive a meaningful macroscopic limit we introduce the diffusive scaling (see, e.g. [6]) for the equation

$$
\left(\partial_{t}+v_{x} \partial_{x}\right) \phi=C F^{-1} \phi
$$

It consists in replacing the macroscopic variables $t$ and $x$ with $\epsilon^{-2} t$ and $\epsilon^{-1} x$ and leads to the rescaled equation

$$
\left(\partial_{t}+\epsilon^{-1} v_{x} \partial_{x}\right) \phi=\epsilon^{-2} C F^{-1} \phi
$$

Formally this is equivalent to replacing the space $\mathcal{V}$ of microscopic velocities with $\epsilon^{-1} \mathcal{V}$ and scaling up the collision frequency by a factor $\epsilon^{-2}$. This is the approach which we take here.

Replacing $\mathbf{v}_{i}$ with $\mathbf{w}_{i}=\epsilon^{-1} \mathbf{v}_{i}$ requires to change the Maxwellians $\mathbf{f}_{\delta}=\left(\exp \left(-\left|\mathbf{v}_{i}-\delta \mathbf{e}_{x}\right|^{2} / 2 \Theta\right), i=1 \ldots N\right)^{T}$ to $\mathbf{f}_{\delta}^{(\epsilon)}=\left(\exp \left(-\left|\mathbf{w}_{i}-\delta \mathbf{e}_{x}\right|^{2} / 2 T\right), i=1 \ldots N\right)^{T}=\left(\exp \left(-\left|\mathbf{v}-\epsilon \delta \mathbf{e}_{x}\right|^{2} / 2 \epsilon^{2} T\right), i=1 \ldots N\right)^{T}$ (leaving the macroscopic bulk velocity $\overline{\mathbf{v}}$ unchanged) which itself makes only sense if we rescale the temperature as $T=\epsilon^{-2} \Theta$. From now on we define

$$
\mathbf{f}_{\delta}^{(\epsilon)}=\mathbf{f}_{\epsilon \delta}^{(1)}=\left(\exp \left(-\left|\mathbf{v}-\epsilon \delta \mathbf{e}_{x}\right|^{2} / 2 \Theta\right), \mathbf{v} \in \mathcal{V}\right)^{T}
$$

with $\Theta>0$ constant. We write $\delta=: \Theta \bar{w}$.

Associated to $\mathbf{f}_{\Theta \bar{w}}^{(\epsilon)}$ are the moments 


$$
\begin{array}{ll}
\text { density } & \rho_{\Theta \bar{w}}^{(\epsilon)}=\left\langle\mathbb{1}, \mathbf{f}_{0}^{(1)}\right\rangle+\mathcal{O}\left(\epsilon^{2}\right) \doteq\left\langle\mathbb{1}, \mathbf{f}_{0}^{(1)}\right\rangle \\
\text { flux } & \phi_{\Theta \bar{w}}^{(\epsilon)}=\left\langle\mathbf{w}_{x}, \mathbf{f}_{\Theta \bar{w}}^{(\epsilon)}\right\rangle=\bar{w} \cdot\left\langle\mathbf{v}_{x}^{2}, \mathbf{f}_{0}^{(1)}\right\rangle+\mathcal{O}\left(\epsilon^{2}\right) \doteq \bar{w} \cdot\left\langle\mathbf{v}_{x}^{2}, \mathbf{f}_{0}^{(1)}\right\rangle
\end{array}
$$

while temperature $T$ given by $\rho T \sim\left\langle|\mathbf{w}|^{2} \mathbf{f}_{\Theta \bar{w}}^{(\epsilon)}\right\rangle \nearrow \infty$

The rescaled steady problem reads

$$
\partial_{x} \phi=\epsilon^{-1} L_{\Theta \bar{w}}^{(\epsilon)} \phi=\epsilon^{-1} L_{\epsilon \Theta \bar{w}}^{(1)} \phi
$$

Repeating the above calculations for the rescaled problem leads to the following result which only concerns the $T_{y}$-even solutions.

Theorem: (a) For $\epsilon \searrow 0$, the general $T_{y}$-even solution of the ODE system takes the form

$$
\begin{aligned}
\phi(x)= & c_{4} \cdot\left(\epsilon \cdot \exp (\bar{w} \lambda x) \mathbf{t}_{4}^{0}+\mathcal{O}\left(\epsilon^{2}\right)+\frac{\exp (\bar{w} \lambda x)-1}{\bar{w} \lambda} \cdot \mathbf{t}_{3}^{\epsilon \Theta \bar{w}}\right) \\
& +\exp \left((1+x) \epsilon^{-1} \Lambda_{\bar{w}}^{+}\right) \phi_{+}(-1)+\exp \left((1-x) \epsilon^{-1} \Lambda_{\bar{w}}^{-}\right) \phi_{-}(+1) \\
\approx & c_{4} \cdot\left(\epsilon(1+\bar{w} \lambda x) \mathbf{t}_{4}^{0}+x \cdot\left[\mathbf{t}_{3}^{0}+\epsilon \bar{w} V_{x}\left(\mathbf{t}_{3}^{0}+\mu \mathbf{f}_{0}\right)\right]\right), \quad \text { for }|x| \ll 1
\end{aligned}
$$

$\mathbf{t}_{i}^{0}, \lambda, \mu$ are the quantities of the preceding section.

(b) Let $\mathbf{n}=\left(n_{x}, n_{y}\right)$ be a multiindex and $\mathbf{v}^{\mathbf{n}}=\mathbf{v}_{x}^{n_{x}} \mathbf{v}_{y}^{n_{y}}$ the $\mathbf{n}$-th moment vector. In the limit $\epsilon=0$, the macroscopic moment is given in first order of $\bar{w}$ as

$$
\left\langle\mathbf{w}^{\mathbf{n}}, \phi\right\rangle=c_{4} \epsilon^{|\mathbf{n}|} \cdot\left\{\begin{array}{cl}
x \cdot\left\langle\mathbf{v}^{\mathbf{n}}, \mathbf{t}_{3}^{0}\right\rangle & n_{x} \text { even } \\
\epsilon\left\langle\mathbf{v}^{\mathbf{n}}, \mathbf{t}_{4}^{0}\right\rangle+\epsilon \bar{w} \lambda x\left\langle\mathbf{v}^{\mathbf{n}}, \mathbf{t}_{4}^{0}+V_{x} \mathbf{t}_{3}^{0}+\mu V_{x} \mathbf{f}_{0}\right\rangle & n_{x} \text { odd }
\end{array}\right.
$$

From this follow easily closure relations betwen moments and their fluxes. For example, the heat coefficient (as the ratio between heat flux and temperature gradient) is given as

$$
q=\left\langle\mathbf{v}_{x}|\mathbf{v}|^{2}, \mathbf{t}_{\mathbf{4}}^{\mathbf{0}}\right\rangle /\left\langle|\mathbf{v}|^{2}, \mathbf{t}_{\mathbf{3}}^{\mathbf{0}}\right\rangle
$$

The above theory is easily extendable to related situations like the following example.

Example: The system

$$
v_{x} \partial_{x} \mathbf{g}=J[\mathbf{f}, \mathbf{g}], \quad v_{x} \partial_{x} \mathbf{h}=J[\mathbf{f}, \mathbf{h}], \quad \mathbf{f}=\mathbf{g}+\mathbf{h}
$$

describes a binary gas mixture of two mechanically identical species $A$ and $B$. Suppose $A$ represents vapor which can evaporate or condensate at the walls, while $B$ is totally reflected thus producing zero flux between the wall. Introducing a pressure difference between the walls induces a flow of $A$ from one wall to the other. Classical asymptotic analysis produces an anomaly ("ghost effect") in the form of infinitesimally thin boundary layers of species $B$ completely stopping the flow of $A[7,8]$. Instead, the above scaling procedure leads to a boundary layer of finite thickness slowing down the flow of $A$ depending on the concentration of $B$. The reason for the differences lies in the fact that the formal asymptotic expansion procedure in $[7,8]$ produces the wrong equilibrium state since it is not capable of the arising bifurcation mode. For details, see [9]. 


\section{CONCLUSIONS}

We have investigated symmetric DVMs at small Mach numbers. A typical feature is a bifurcation phenomenon of the steady linearized transport operator at zero bulk velocity. A detailed analysis allows to derive explicit formulas for the closure relations of the moment system in the hydrodynamic limit.

\section{REFERENCES}

[1] F. Rogier, J. Schneider. A direct method for solving the Boltzmann equation. Transp. Theory Stat. Phys., 23:313-338, 1994.

[2] A. Palczewski, J. Schneider and A. V. Bobylev. A consistency result for a discrete velocity model of the Boltzmann equation. SIAM J. Numer. Anal., 34:1865-1883, 1997.

[3] Stefan Brechtken. Classification of Lattice Group Models, High Order Discretizations of Boltzmann's Collision Operator and Parallelization. Dissertation, Technische Universität Ilmenau, 2015.

[4] H. Babovsky. Discrete kinetic models in the fluid dynamic limit. Computers and Mathematics with Applications, 67:256-271, 2014.

[5] H. Babovsky and T. Płatkowski, Kinetic boundary layers for the Boltzmann equation on discrete velocity lattices. Arch. Mech., 60:87-116, 2003

[6] H. Babovsky and P. Kowalczyk. Diffusion limits for discrete velocity models in a thin gap. Multiscale Modelling and Simulation, 6:631-655, 2007.

[7] S. Takata and K. Aoki. The ghost effect in the continuum limit for a vapor-gas mixture around condensed phases: Asymptotic analysis of the Boltzmann equation. Transport Theor. Statist. Phys., 30:205-237, 2001.

[8] K. Aoki, S. Takata, and S. Taguchi. Vapor flows with evaporation and condensation in the continuum limit: effect of a trace of noncondensable gas. Eur. Journ. Mech. B/Fluids, 22:51-71, 2003.

[9] Hans Babovsky. Macroscopic limit for an evaporation-condensation prolem. Preprint, Technische Universität Ilmenau, 2015, submitted. 